\title{
Fatores preditivos para o desenvolvimento da síndrome cardiorrenal em pacientes com insuficiência cardíaca
}

\author{
Predictive factors for the development of cardiorenal syndrome in patients with heart failure \\ Factores predictivos para el desarrollo del síndrome cardiorrenal en pacientes con insuficiencia
}

cardíaca

Recebido: 18/01/2021 | Revisado: 23/01/2021 | Aceito: 26/01/2021 | Publicado: 03/02/2021

\author{
Alexandre Henrique Costa Gonçalves \\ ORCID: https://orcid.org/0000-0003-0797-7722 \\ Centro Universitário de Patos, Brasil \\ E-mail: alexandregoncalves@med.fiponline.edu.br \\ Milena Nunes Alves de Sousa \\ ORCID: https://orcid.org/0000-0002-9431-6326 \\ Centro Universitário de Patos, Brasil \\ E-mail: milenanunes@ fiponline.edu.br \\ Tiago Bruno Carneiro de Farias \\ ORCID: https://orcid.org/0000-0002-0473-1244 \\ Centro Universitário de Patos, Brasil \\ E-mail: tiagofarias@ fiponline.edu.br
}

\begin{abstract}
Resumo
Objetivo: analisar, a partir de uma revisão sistemática da literatura, quais são os fatores preditivos para o desenvolvimento da síndrome cardiorrenal em pacientes com insuficiência cardíaca. Métodos: efetuou-se uma revisão sistemática da literatura a partir da questão de pesquisa "Quais os fatores preditivos para o desenvolvimento da síndrome cardiorrenal em pacientes com insuficiência cardíaca?”. Para a seleção dos artigos que compõem este estudo, realizou-se uma busca nas seguintes bases de dados: Medical Publisher (PUBMED), Biblioteca Virtual em Saúde (BVS), Science Direct (SD) e Semantic Scholar utilizando-se como critérios de elegibilidade apenas estudos observacionais ou ensaios clínicos randomizados, que contemplassem a questão de pesquisa, que fossem relevantes e atuais para abordar o tema. A amostra final é composta por 25 publicações. Resultados: os principais fatores preditivos para o desenvolvimento da síndrome cardiorrenal se associam com os fatores de risco cardiovasculares e às manifestações geradas a partir do comprometimento renal. Conclusão: a junção de comorbidades do paciente, com fatores de risco para desenvolvimento de doença cardiovascular, de injúria renal aguda associado com marcadores laboratoriais de injúria miocárdica e de lesão renal podem atuar como fatores preditivos confiáveis para o desenvolvimento da síndrome cardiorrenal.
\end{abstract}

Palavras-chave: Síndrome cardiorrenal; Fatores preditivos; Insuficiência cardíaca; Disfunção renal.

\begin{abstract}
Objective: to analyze, from a systematic review of the literature, what are the predictive factors for the development of cardiorrenal syndrome in patients with heart failure. Methods: a systematic review of the literature was performed from the research question "What are the predictive factors for the development of cardiorrenal syndrome in patients with heart failure? For the selection of the articles that compose this study, a search was made in the following databases: Medical Publisher (PUBMED), Virtual Health Library (VHL), Science Direct (SD) and Semantic Scholar, using as eligibility criteria only observational studies or randomized clinical trials that contemplated the research question, which were relevant and current to address the theme. The final sample is composed of 25 publications. Results: the main predictive factors for the development of cardiorrenal syndrome are associated with cardiovascular risk factors and manifestations generated from renal impairment. Conclusion: The junction of comorbidities of the patient, with risk factors for the development of cardiovascular disease, acute renal injury associated with laboratory markers of myocardial injury and renal injury can act as reliable predictive factors for the development of the cardiorrenal syndrome.
\end{abstract}

Keywords: Cardiorenal syndrome; Predictive factors; Heart failure; Renal dysfunction.

\section{Resumen}

Objetivo: Analizar, a partir de una revisión sistemática de la literatura, cuáles son los factores predictivos para el desarrollo del síndrome cardiorrenal en pacientes con insuficiencia cardíaca. Métodos: Se realizó una revisión sistemática de la literatura a partir de la pregunta de investigación "¿Cuáles son los factores predictivos del desarrollo 
del síndrome cardiorrenal en pacientes con insuficiencia cardíaca? Para seleccionar los artículos que componen este estudio, se realizó una búsqueda en las siguientes bases de datos: Medical Publisher (PUBMED), Virtual Health Library (BVS), Science Direct (SD) y Semantic Scholar, utilizando como criterio de elegibilidad sólo estudios observacionales o ensayos clínicos aleatorizados que contemplaran la pregunta de investigación, que fueran relevantes y actuales para abordar el tema. La muestra final está compuesta por 25 publicaciones. Resultados: los principales factores predictivos para el desarrollo del síndrome cardiorrenal se asocian a los factores de riesgo cardiovascular y a las manifestaciones generadas a partir del deterioro renal. Conclusión: La unión de las comorbilidades del paciente con los factores de riesgo para el desarrollo de enfermedad cardiovascular, la lesión renal aguda asociada a los marcadores de laboratorio de lesión miocárdica y la lesión renal pueden actuar como factores predictivos fiables para el desarrollo del síndrome cardiorrenal.

Palabras clave: Síndrome cardiorrenal; Factores predictivos; Insuficiencia cardíaca; Disfunción renal.

\section{Introdução}

Os sistemas cardiovascular, renal e vascular-endotelial trabalham juntos em função de manter o tônus vascular, volume sanguíneo e estabilidade hemodinâmica adequados para manter o organismo em bom funcionamento e estão, portanto, inevitavelmente interconectados em estados de distúrbios no organismo. A relação bidirecional e sinérgica entre o coração, os rins e os vasos sanguíneos, na qual falhas em um órgão pode provocar mudanças patológicas no outro é denominada, atualmente, síndrome cardiorrenal. Esta, ocorre na presença de disfunção vascular, causada por hipertensão, sobrecarga de volume e congestão venosa (Savira et al., 2020).

Tendo em vista os vasos sanguíneos compartilhados entre os sistemas cardíaco e renal, a disfunção em um órgão se torna um fator de risco para gerar disfunção no outro. Os fatores de risco renais, tais como doença renal crônica, distúrbios hidroeletrolíticos, elevação dos níveis de creatinina, hipercalemia e insuficiência renal aguda, possuem forte impacto em reduzir as funções cardíacas. Da mesma forma, fatores de risco cardiovasculares, tais como hipertensão arterial sistêmica, doença arterial coronariana, obesidade, diabetes nellitus, fração de ejeção reduzida, edema agudo de pulmão, histórico de insuficiência cardíaca, fibrilação atrial e aterosclerose podem resultar na piora da função renal (Correia et al., 2020; Raina et al., 2020).

Doenças cardíacas e renais compartilham fatores de riscos vasculares em comum, tais como hipertensão arterial sistêmica (HAS) e diabetes mellitus (DM). A sobreposição de fatores de risco pode ser observada em várias interações hemodinâmicas em injúrias cardíacas e renais, interação neuro-hormonal, doença renal crônica e doenças ateroscleróticas. Doença renal e insuficiência cardíaca concomitantes estão diretamente relacionadas e são um importante fator indicativo de pior prognóstico, levando a um grande agravo no sistema de saúde e na qualidade de vida dos pacientes acometidos (Andrade et al., 2014; Melo, Bezerra \& Sousa, 2014; Dantas et al., 2020; Raina et al., 2020).

Os rins, órgãos bastante vascularizados, sofrem com hipoperfusão tecidual e deixa de realizar suas funções de forma eficaz quando o coração deixa de bombear o sangue adequadamente. Da mesma forma, o mau funcionamento renal pode resultar em elevação da pressão arterial, aumentando a carga de trabalho do coração e contribuindo para disfunções cardíacas. Doença renal e cardíaca frequentemente coexistem no mesmo paciente em estados agudos ou crônicos (Jentzer et al., 2020).

Autores corroboram com a assertiva anterior ao afirmarem que há forte associação entre a piora da função renal e a mortalidade em pacientes com insuficiência cardíaca aguda descompensada. Considerando essa comunicação biológica complexa e bidirecional entre esses dois órgãos, tais alterações no organismo têm sido descritas como síndrome cardiorrenal (Legrand et al., 2014).

O Consenso da Iniciativa de Qualidade de Diálise Aguda formalizou uma definição para o termo síndrome cardiorrenal em 2010. Esse sistema de classificação divide a condição clínica em cinco tipos diferentes conforme a disfunção e cronicidade no órgão primário afetado (Savira et al., 2020). 
Assim, identificou-se que além da disfunção contrátil cardíaca e da sobrecarga de volume resultante, os mecanismos que geram congestão venosa e os mecanismos neuro-hormonais também estão envolvidos no desenvolvimento desse distúrbio. Essa nova classificação possibilitou um avanço para um melhor entendimento da fisiopatologia e das estratégias terapêuticas das interações bidirecionais entre o coração e os rins (Uduman, 2018).

Apesar das manifestações clínicas comuns dessa síndrome, tais como hipertensão arterial sistêmica, aterosclerose, sintomas de insuficiência cardíaca, tais como cansaço, fadiga, intolerância ao exercício, ortopneia e dispneia paroxística noturna, doença renal crônica - associada com cardiomiopatia, a qual se refere a um conjunto de distúrbios envolvendo o coração e os rins, em que a disfunção aguda ou crônica em um desses órgãos pode induzir disfunção aguda ou crônica no outro (Rangaswami \& Mathew, 2018; Chimada et al., 2020).

Essa síndrome é dividida ainda em dois grupos, cardiorrenal e renocardíaco, com base na injúria primária do órgão (coração ou rins), podendo ser enquadradas em distúrbios agudos ou crônicos. Os tipos mais frequentemente identificados na prática clínica e bem descritos na literatura são a síndrome cardiorrenal tipo I e tipo II. Os outros três tipos estão relacionados com definição conceitual, não sendo muito comuns na prática clínica e possuem diferentes características fisiopatológicas, apesar da abordagem terapêutica ser semelhante (Lullo et al., 2017).

A síndrome cardiorrenal tipo I é caracterizada pela piora aguda da função cardíaca, resultando em injúria renal aguda e é mais comum nos pacientes com insuficiência cardíaca descompensada. A injúria renal aguda é um fator de risco de mortalidade independente em casos agudos de insuficiência cardíaca descompensada, incluindo os casos de infarto agudo do miocárdio (IAM) e em pacientes com ventrículo esquerdo com fração de ejeção reduzida (Lullo et al., 2017; Borges, Figueiredo \& Sousa, 2020; Raina et al., 2020).

A alteração e piora da função renal são fatores de risco bem conhecidos associados com a piora do prognóstico de pacientes com insuficiência cardíaca aguda descompensada. A síndrome cardiorrenal surge da combinação de alterações sistêmicas e hemodinâmicas intra-renais, além dos mecanismos inflamatórios envolvidos nas lesões renais ou cardíacas (Legrand et al., 2014).

A anemia é um importante fator preditor de mortalidade no estabelecimento da síndrome cardiorrenal sendo atribuída ao defeito na eritropoiese e diminuição na biodisponibilidade de ferro sérico devido à redução na função renal. Isso leva a isquemia e vasodilatação periférica com a subsequente hiperativação do sistema renina-angiotensina-aldosterona e do sistema nervoso simpático, sobrecarga de volume e congestão venosa. (Yogasundaram, Chappell, Braam \& Oudit, 2019; Savira et al., 2020).

Em pacientes com doença renal crônica, a anemia está relacionada com deficiência cognitiva, piora da qualidade de vida, progressão da doença renal, comorbidades cardiovasculares e aumento da mortalidade (Kumar, Wettersten \& Garimella 2019).

O tipo II é caracterizado pelo início do desenvolvimento de doença renal crônica em pacientes com insuficiência cardíaca. Acredita-se que ambos os distúrbios coexistem nos pacientes e a condição cardíaca é subjacente à ocorrência ou progressão da lesão renal crônica. Dentre os mecanismos fisiopatológicos mais importantes dessa condição, destacam-se a ativação neuro-hormonal, hipoperfusão e congestão venosa, inflamação, aterosclerose e estresse oxidativo. É mais comum em pacientes com insuficiência cardíaca crônica (Lullo et al., 2017; Uduman, 2018; Raina et al., 2020).

Assim, o presente estudo tem como objetivo analisar, a partir de uma revisão sistemática da literatura, quais são os fatores preditivos para o desenvolvimento da síndrome cardiorrenal em pacientes com insuficiência cardíaca. Dentre as razões para justificar a realização desse tipo de estudo, importante em temáticas atuais e ainda pouco abordadas, destaca-se a possibilidade de contribuir com as práticas clínicas e o meio científico, fornecendo evidências científicas integradas e relevantes para a prática clínica. 


\section{Metodologia}

Uma revisão sistemática é um tipo de estudo da literatura feita a partir da revisão de uma questão de pesquisa claramente formulada que usa métodos sistemáticos e explícitos com o intuito de identificar, selecionar e avaliar criticamente pesquisas relevantes, além de coletar e analisar dados dos estudos incluídos na revisão. A natureza desse tipo de revisão significa que ela tende a ter uma qualidade superior aos demais tipos de estudo, como revisão integrativa ou bibliométrica, mais compreensível e menos tendenciosa que outros tipos de revisão da literatura. Quando bem realizada e bem estruturada, a revisão sistemática pode contribuir significativamente para a construção do conhecimento (Siddaway, Wood \& Hedges, 2019).

No intuito de responder à questão de pesquisa, efetuou-se uma revisão sistemática cujo objetivo é uma revisão que visa à integração da pesquisa qualitativa. Um dos passos iniciais para a realização desse tipo de revisão é optar por se será feito uma análise quantitativa ou qualitativa. Há dois tipos de síntese de pesquisa qualitativa: uma revisão narrativa e a meta-síntese, que consiste na síntese de estudos qualitativos sobre um tópico a fim de identificar e localizar temas, conceitos ou teorias para que possam fornecer informações e explicações novas e atualizadas para o fenômeno que se está analisando. No presente estudo, optou-se por uma análise qualitativa e uma meta-síntese (Siddaway, Wood \& Hedges, 2019).

As revisões sistemáticas seguem protocolos específicos com o objetivo de contribuir para que as revisões da literatura ganhem em qualidade de pesquisa. Assim, seguiram-se os seguintes passos para a confecção do presente estudo (Galvão \& Ricarte, 2019):

1 - Delimitação da questão de pesquisa a ser tratada na revisão: nessa fase, foi estabelecida a questão norteadora da pesquisa, $\mathrm{P}=$ População (pacientes com insuficiência cardíaca), $\mathrm{E}=$ Exposição de interesse $($ síndrome cardiorrenal) e $\mathrm{O}=$ Desfecho (fatores preditivos para o desenvolvimento da síndrome cardiorrenal). Dessa forma, o questionamento traçado foi “quais os fatores preditivos para o desenvolvimento da síndrome cardiorrenal em pacientes com insuficiência cardíaca?”.

2 - Estratégias de busca empregadas em cada base de dados para consulta e coleta de material: realizada entre agosto e setembro de 2020, em que se utilizaram as bases de dados da Biblioteca Virtual em Saúde (BVS), Medical Publisher (PUBMED) e Science Direct para pesquisar estudos que abordassem a questão da revisão. Essas plataformas foram selecionadas por serem de amplo e fácil acesso, de simples uso, intuitivas e pelo grande número de publicações de acesso livre e gratuito.

3 - Processo de seleção dos artigos científicos - elaboração de critérios para busca avançada: utilizaram-se os seguintes Descritores em Ciências da Saúde (DeCs) em inglês "cardiorrenal syndrome" e "heart failure" unidos pelo operador booleano "AND". Essa associação se aplicou em todas as bases de dados da literatura selecionadas. Além disso, incluíram-se estudos observacionais ou de coorte, ensaios clínicos randomizados, textos disponíveis completos e publicados entre 2017 e 2020, uma vez que o intuito é o de selecionar estudos mais atuais e com as informações mais atualizadas. Os artigos repetidos em mais de uma base de dados foram desconsiderados na contagem e não fazem parte da amostra final. Esse processo de seleção deve ser explicitado na forma de fluxo, conforme as diretrizes da Preferred Reporting Items for Systematic Reviews and Meta-Analyses ou Recomendação PRISMA e está demonstrada no Fluxograma 1. 
Fluxograma 1. Etapas seguidas para seleção dos artigos que compõem esse estudo conforme recomendação PRISMA.

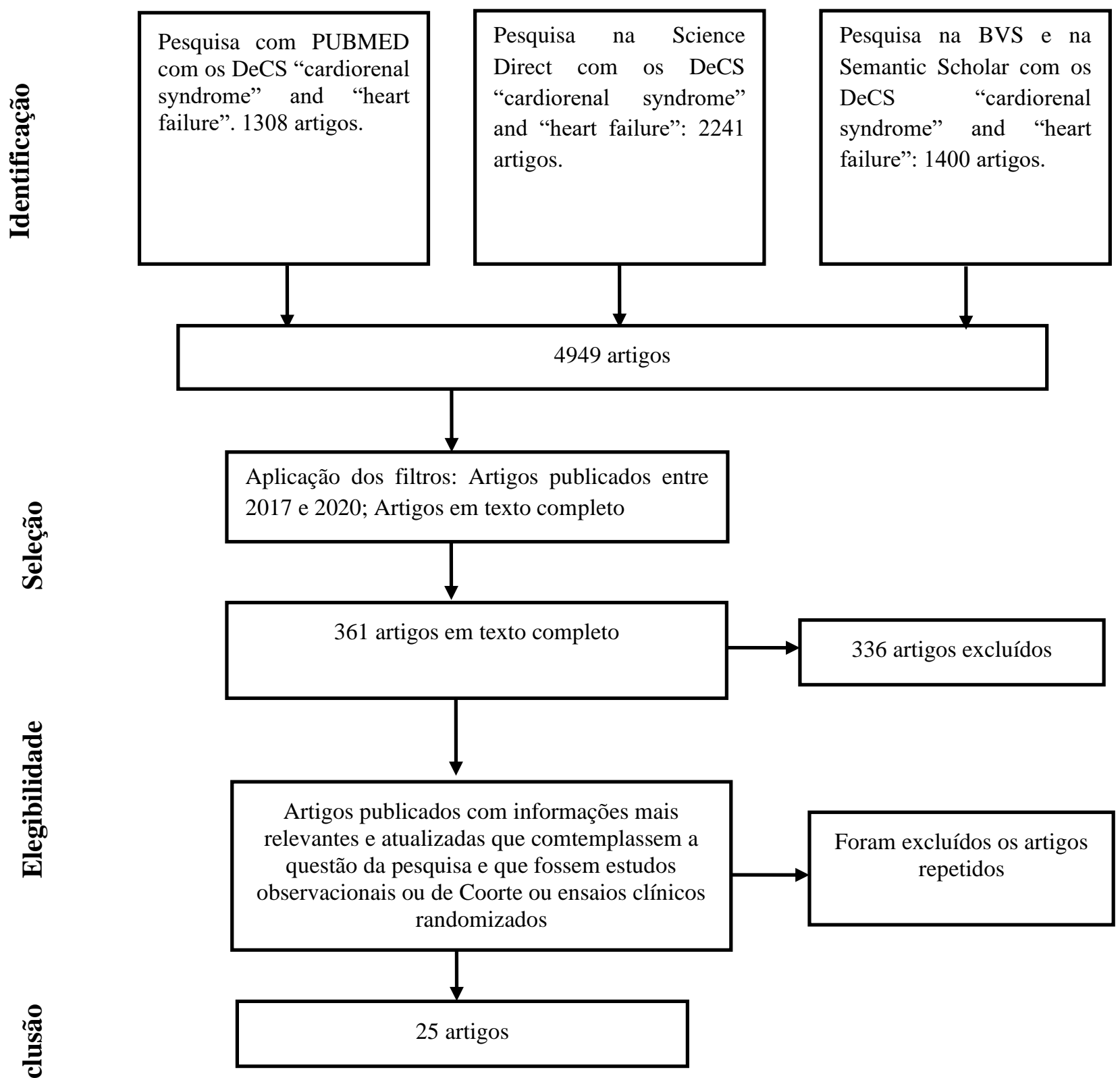

Fonte: Autores (2020).

4 - Análise criteriosa dos artigos selecionados - seleção de texto: buscaram-se informações relacionadas com os fatores preditivos para o desenvolvimento da síndrome cardiorrenal em pacientes já diagnosticados com insuficiência cardíaca e, a partir de então, efetivou-se a síntese dos dados em quadros. Esta fase realizou-se com o autor do presente estudo e seu orientador, sendo que qualquer incongruência entre os estudos selecionados foi solucionada mediante discussões sobre o assunto até chegar a um consenso sobre as informações encontradas.

5 - Sistematização das informações encontradas: o objetivo do estudo é analisar, a partir da revisão sistemática qualitativa, meta-síntese, da literatura, os fatores preditivos para o desenvolvimento da síndrome cardiorrenal em pacientes com insuficiência cardíaca. Portanto, nesta fase apresentam-se os achados principais da investigação, selecionando-se variáveis como: ano, tipo de estudo, base de dados, país, frequência, diagnóstico, manifestações clínicas, fatores de risco e fatores 
prognósticos desse distúrbio. Assim, as informações obtidas foram sintetizadas no Quadro 2 no qual é evidenciada a extração dos resultados que respondem à questão de pesquisa, para que seja possível a análise e síntese dos estudos.

Após esses passos, é necessário atentar para a qualidade metodológica e de evidência dos estudos, com o intuito de classificar o nível de evidência dos estudos selecionados pelos 5 passos anteriores. Para isso seguiu-se os seguintes passos:

1 - Avaliação da qualidade metodológica: para classificar a qualidade metodológica dos estudos (Quadros 3 e 4) que compõem esse artigo (Figura 1) utilizaram-se as Diretrizes Metodológicas do Sistema GRADE (Brasil, 2014) em que o percurso de filtragem também adotou a Preferred Reporting Items for Systematic Reviews and Meta-Analyses ou a Recomendação PRISMA (Galvão, Pansani \& Harrad, 2015).

2 - Avaliação da qualidade de evidência dos estudos selecionados: o Sistema GRADE (Brasil, 2014) foi utilizado para graduar a qualidade das evidências e a força das recomendações. De acordo com as Diretrizes Metodológicas do Sistema GRADE, estudos observacionais devem iniciar com classificação baixa e, a partir de determinados critérios, é possível aumentar a qualidade da evidência. Da mesma forma, ensaios clínicos randomizados iniciam com classificação alta e, a partir de certos critérios, pode-se diminuir ou manter a qualidade da evidência. Assim, as informações mensuradas estão demonstradas nos Quadros 3 e 4.

De acordo com essa diretriz, ensaios clínicos randomizados iniciam com nível de evidência alto e a partir da identificação dos fatores responsáveis pela redução no nível de evidência: limitações metodológicas (risco de viés); inconsistência; evidência indireta; imprecisão; viés de publicação, deve-se reduzir o nível de evidência. Caso o nível não seja rebaixado pela identificação dos critérios mencionados, a qualidade da evidência desses estudos permanece como alta.

Em relação aos estudos observacionais, devem iniciar com nível de evidência baixo e a partir da presença de três fatores principais: grande magnitude de efeito; gradiente dose-resposta; fatores de confusão residuais, os quais aumentam a confiança na estimativa, os estudos observacionais devem ter seu nível de evidência aumentado.

A avaliação da presença de fatores que diminuem o nível de evidência dos estudos selecionados é necessária pois, a partir do momento em que há limitações metodológicas ocorre uma redução na confiança da estimativa de efeito desses estudos.

A classificação do nível de evidência dos estudos que compõem uma revisão sistemática é essencial tendo em vista que ele representa a qualidade da evidência científica disponível e define a confiança na informação utilizada, possibilitando a definição de uma determinada recomendação, além de disseminar informações importantes sobre determinado tema relevante para o contexto atual.

Assim, os estudos selecionados foram analisados em suas metodologias em busca da presença ou ausência desses critérios, no intuito de se estabelecer o nível de evidência de cada um dos estudos e classificá-los, de acordo com o sistema GRADE em: alto, moderado, baixo e muito baixo. Para realizar essa classificação o sistema GRADE analisa fatores que diminuem o nível de evidência, utilizados para ensaios clínicos randomizados e fatores que aumentam o nível de evidência, avaliados em estudos observacionais.

A etapa final da confecção dessa revisão sistemática constitui-se pela apresentação dos resultados, os quais foram sintetizados, constituindo-se neste documento. Reforça-se que os resultados foram apresentados em forma de síntese narrativa, caracterizada pela análise descritiva dos dados quantitativos.

\section{Resultados}

De acordo com os dados presentes no Quadro 1, pode-se inferir que os estudos selecionados $(\mathrm{N}=25)$ foram escolhidos dentre as seguintes bases de dados: Medical Publisher (PUBMED N=10), Biblioteca Virtual em Saúde (N=9), Science Direct 
$(\mathrm{N}=4)$ e Semantic Scholar $(\mathrm{N}=2)$. Além disso, a maior parte dos estudos selecionados caracteriza-se como Estudo de Coorte $(\mathrm{N}=19)$, seguidos por um total de $(\mathrm{N}=5)$ Ensaios clínicos randomizados e apenas $(\mathrm{N}=1)$ Estudo de caso-controle.

Em relação aos países, há 18 estudos de diferentes países, ressaltando-se a heterogeneidade de origem da publicação dos estudos selecionados, bem como para a relevância atual da síndrome cardiorrenal, tendo em vista a quantidade de países diferentes publicando sobre a temática.

Quadro 1. Caracterização dos artigos de acordo com autores e ano de publicação, título, tipo de estudo, base de dados de publicação e país de origem.

\begin{tabular}{|c|c|c|c|c|}
\hline Autores (ano) & Título do artigo & Tipo de estudo & $\begin{array}{l}\text { Base de } \\
\text { Dados }\end{array}$ & País \\
\hline $\begin{array}{l}\text { Acle et al. } \\
(2018)\end{array}$ & $\begin{array}{l}\text { Renal function in outpatients with heart failure } \\
\text { with reduced ejection fraction; analysis of its } \\
\text { evolution and forecast implications. A } 4 \text { year } \\
\text { follow up in a Multidisciplinary Unit of Heart } \\
\text { Failure. }\end{array}$ & $\begin{array}{l}\text { Estudo de Coorte } \\
\text { retrospectivo }\end{array}$ & BVS & Uruguai \\
\hline $\begin{array}{l}\text { Al-Hwiesh et al. } \\
\text { (2019) }\end{array}$ & $\begin{array}{l}\text { Tidal peritoneal dialysis versus ultrafiltration in } \\
\text { type } 1 \text { cardiorenal syndrome: A prospective } \\
\text { randomized study }\end{array}$ & $\begin{array}{l}\text { Ensaio clínico } \\
\text { randomizado }\end{array}$ & BVS & Arábia Saudita \\
\hline $\begin{array}{l}\text { Al-Jarallah et } \\
\text { al. (2018) }\end{array}$ & $\begin{array}{l}\text { Incidence and impact of cardiorenal anaemia } \\
\text { syndrome on all-cause mortality in acute heart } \\
\text { failure patients stratified by left ventricular } \\
\text { ejection fraction in the Middle East }\end{array}$ & $\begin{array}{l}\text { Estudo de Coorte } \\
\text { retrospectivo }\end{array}$ & PUBMED & Kuwait \\
\hline $\begin{array}{l}\text { Dos Reis et al. } \\
(2019)\end{array}$ & $\begin{array}{l}\text { Impact of renal dysfunction on the management } \\
\text { and outcome of acute heart failure: results from } \\
\text { the French prospective, multicentre, DeFSSICA } \\
\text { survey }\end{array}$ & $\begin{array}{c}\text { Estudo de Coorte } \\
\text { prospectivo }\end{array}$ & BVS & França \\
\hline $\begin{array}{l}\text { Inaguma et al. } \\
(2017)\end{array}$ & $\begin{array}{l}\text { Relationship between history of coronary heart } \\
\text { disease at dialysis initiation and onset of events } \\
\text { associated with heart disease: a propensity- } \\
\text { matched analysis of a prospective cohort study }\end{array}$ & $\begin{array}{c}\text { Estudo de Coorte } \\
\text { prospectivo }\end{array}$ & PUBMED & Japão \\
\hline $\begin{array}{l}\text { Lannemyr et al. } \\
\text { (2018) }\end{array}$ & $\begin{array}{l}\text { Differential Effects of Levosimendan and } \\
\text { Dobutamine on Glomerular Filtration Rate in } \\
\text { Patients With Heart Failure and Renal } \\
\text { Impairment: A Randomized Double-Blind } \\
\text { Controlled Trial }\end{array}$ & $\begin{array}{l}\text { Ensaio clínico } \\
\text { randomizado }\end{array}$ & BVS & Suécia \\
\hline $\begin{array}{l}\text { Pallangyo et al. } \\
\text { (2017) }\end{array}$ & $\begin{array}{l}\text { Cardiorenal Anemia Syndrome and Survival } \\
\text { among Heart Failure Patients in Tanzania: A } \\
\text { Prospective Cohort Study }\end{array}$ & $\begin{array}{c}\text { Estudo de Coorte } \\
\text { prospectivo }\end{array}$ & BVS & Tanzânia \\
\hline $\begin{array}{l}\text { Premuzic et al. } \\
\text { (2017) }\end{array}$ & $\begin{array}{l}\text { Continuous Veno-Venous Hemofiltration } \\
\text { Improves Survival of Patients With Congestive } \\
\text { Heart Failure and Cardiorenal Syndrome } \\
\text { Compared to Slow Continuous Ultrafiltration }\end{array}$ & $\begin{array}{c}\text { Estudo de Coorte } \\
\text { prospectivo }\end{array}$ & BVS & Croácia \\
\hline $\begin{array}{l}\text { Verbrugge et } \\
\text { al. (2018) }\end{array}$ & $\begin{array}{l}\text { Spironolactone to increase natriuresis in } \\
\text { congestive heart failure with cardiorrenal } \\
\text { syndrome }\end{array}$ & $\begin{array}{c}\text { Estudo de Coorte } \\
\text { prospectivo }\end{array}$ & PUBMED & $\begin{array}{l}\text { Estados } \\
\text { Unidos da } \\
\text { América }\end{array}$ \\
\hline
\end{tabular}




\begin{tabular}{|c|c|c|c|c|}
\hline $\begin{array}{l}\text { Grodin et al. } \\
(2018)\end{array}$ & $\begin{array}{l}\text { Direct comparison of ultrafiltration to } \\
\text { pharmacological decongestion in heart failure: a } \\
\text { per-protocol analysis of CARRESS-HF }\end{array}$ & $\begin{array}{l}\text { Estudo de Coorte } \\
\text { retrospectivo }\end{array}$ & PUBMED & $\begin{array}{l}\text { Estados } \\
\text { Unidos da } \\
\text { América }\end{array}$ \\
\hline $\begin{array}{l}\text { Grossekettler et } \\
\text { al. (2019) }\end{array}$ & $\begin{array}{l}\text { Peritoneal dialysis as therapeutic option in heart } \\
\text { failure patients }\end{array}$ & $\begin{array}{l}\text { Estudo de Coorte } \\
\text { prospectivo }\end{array}$ & PUBMED & Alemanha \\
\hline $\begin{array}{l}\text { Jung, Hae- } \\
\text { Hyuk (2019) }\end{array}$ & $\begin{array}{l}\text { Association of Optimal Blood Pressure With } \\
\text { Critical Cardiorenal Events and Mortality in } \\
\text { High-Risk and Low-Risk Patients Treated With } \\
\text { Antihypertension Medications }\end{array}$ & $\begin{array}{l}\text { Estudo de Coorte } \\
\text { retrospectivo }\end{array}$ & PUBMED & Coreia do Sul \\
\hline Li et al. (2018) & $\begin{array}{l}\text { Is the mean platelet volume a predictive marker } \\
\text { of a high in-hospital mortality of acute } \\
\text { cardiorrenal syndrome patients receiving } \\
\text { continuous renal replacement therapy? }\end{array}$ & $\begin{array}{l}\text { Estudo de Coorte } \\
\text { retrospectivo }\end{array}$ & PUBMED & China \\
\hline $\begin{array}{l}\text { Linhart et al. } \\
(2018)\end{array}$ & $\begin{array}{l}\text { Systemic inflammation in acute cardiorenal } \\
\text { syndrome: an observational pilot study }\end{array}$ & $\begin{array}{l}\text { Estudo de Coorte } \\
\text { transversal }\end{array}$ & PUBMED & Alemanha \\
\hline $\begin{array}{l}\text { Matsushita et } \\
\text { al. (2017) }\end{array}$ & $\begin{array}{l}\text { Kidney Disease Measures and Left Ventricular } \\
\text { Structure and Function: The Atherosclerosis Risk } \\
\text { in Communities Study }\end{array}$ & $\begin{array}{l}\text { Estudo de Coorte } \\
\text { prospectivo }\end{array}$ & PUBMED & $\begin{array}{l}\text { Estados } \\
\text { Unidos da } \\
\text { América }\end{array}$ \\
\hline $\begin{array}{l}\text { Mavrakanas et } \\
\text { al. (2017) }\end{array}$ & $\begin{array}{l}\text { Epidemiology and Natural History of the } \\
\text { Cardiorenal Syndromes in a Cohort with } \\
\text { Echocardiography }\end{array}$ & $\begin{array}{l}\text { Estudo de Coorte } \\
\text { retrospectivo }\end{array}$ & PUBMED & $\begin{array}{l}\text { Estados } \\
\text { Unidos da } \\
\text { América }\end{array}$ \\
\hline $\begin{array}{l}\text { Virzi et al. } \\
(2019)\end{array}$ & $\begin{array}{l}\text { Lipopolysaccharide in systemic circulation } \\
\text { induces activation of inflammatory response and } \\
\text { oxidative stress in cardiorrenal syndrome type } 1\end{array}$ & $\begin{array}{l}\text { Estudo de caso- } \\
\text { controle }\end{array}$ & PUBMED & Itália \\
\hline $\begin{array}{l}\text { Watanabe et al. } \\
\text { (2018) }\end{array}$ & $\begin{array}{l}\text { Prognostic Significance of Serum Indoxyl Sulfate } \\
\text { and Albumin for Patients with Cardiovascular } \\
\text { Disease }\end{array}$ & $\begin{array}{l}\text { Estudo de Coorte } \\
\text { prospectivo }\end{array}$ & PUBMED & Japão \\
\hline $\begin{array}{l}\text { Zhang et al. } \\
\text { (2018) }\end{array}$ & $\begin{array}{l}\text { Combination of Amino-Terminal Pro-BNP, } \\
\text { Estimated GFR, and High-Sensitivity CRP for } \\
\text { Predicting Cardiorenal Syndrome Type } 1 \text { in } \\
\text { Acute Myocardial Infarction Patients }\end{array}$ & $\begin{array}{l}\text { Estudo de Coorte } \\
\text { retrospectivo }\end{array}$ & PUBMED & China \\
\hline $\begin{array}{l}\text { García-Blas et } \\
\text { al. (2017) }\end{array}$ & $\begin{array}{l}\text { Diuretic Strategies in Acute Heart Failure and } \\
\text { Renal Dysfunction: Conventional vs } \\
\text { Carbohydrate Antigen 125-guided Strategy. } \\
\text { Clinical Trial Design }\end{array}$ & $\begin{array}{l}\text { Ensaio clínico } \\
\text { randomizado }\end{array}$ & $\begin{array}{l}\text { Science } \\
\text { Direct }\end{array}$ & Espanha \\
\hline Ng et al. (2017) & $\begin{array}{l}\text { Proenkephalin, Renal Dysfunction, and Prognosis } \\
\text { in Patients With Acute Heart Failure A GREAT } \\
\text { Network Study }\end{array}$ & $\begin{array}{l}\text { Estudo de Coorte } \\
\text { prospectivo }\end{array}$ & $\begin{array}{l}\text { Science } \\
\text { Direct }\end{array}$ & Inglaterra \\
\hline $\begin{array}{l}\text { Siegenthaler et } \\
\text { al. (2017) }\end{array}$ & $\begin{array}{l}\text { Impact of cardio-renal syndrome on adverse } \\
\text { outcomes in patients with Fabry disease in a } \\
\text { long-term follow-up }\end{array}$ & $\begin{array}{l}\text { Estudo de Coorte } \\
\text { prospectivo }\end{array}$ & $\begin{array}{l}\text { Science } \\
\text { Direct }\end{array}$ & Suíça \\
\hline $\begin{array}{l}\text { Suresh et al. } \\
(2017)\end{array}$ & $\begin{array}{l}\text { Cardiorenal syndrome type } 4: \text { A study of } \\
\text { cardiovascular diseases in chronic kidney disease }\end{array}$ & $\begin{array}{l}\text { Estudo de Coorte } \\
\text { prospectivo }\end{array}$ & $\begin{array}{l}\text { Science } \\
\text { Direct }\end{array}$ & Índia \\
\hline $\begin{array}{l}\text { Shao et al. } \\
(2018)\end{array}$ & $\begin{array}{l}\text { Effectiveness and Safety of Peritoneal Dialysis } \\
\text { Treatment in Patients with Refractory Congestive } \\
\text { Heart Failure due to Chronic Cardiorenal } \\
\text { Syndrome }\end{array}$ & $\begin{array}{l}\text { Ensaio clínico } \\
\text { randomizado }\end{array}$ & $\begin{array}{l}\text { Semantic } \\
\text { Scholar }\end{array}$ & China \\
\hline
\end{tabular}




\begin{tabular}{|l|l|l|l|l|c|}
\hline $\begin{array}{l}\text { Robles et al. } \\
\mathbf{( 2 0 2 0 )}\end{array}$ & $\begin{array}{l}\text { Sacubitril-valsartan Improves } \\
\text { Cardiorenal Syndrome (CRS) }\end{array}$ & Anemia of & $\begin{array}{c}\text { Ensaio clínico } \\
\text { randomizado }\end{array}$ & $\begin{array}{c}\text { Semantic } \\
\text { scholar }\end{array}$ & Espanha \\
\hline
\end{tabular}

Fonte: Autores (2020).

Baseando-se na questão de pesquisa: "Em pacientes com insuficiência cardíaca aguda descompensada, quais os fatores preditivos para o desenvolvimento da síndrome cardiorrenal?", investigaram-se os principais resultados encontrados nos estudos selecionados.

Quadro 2. Objetivos dos estudos selecionados e principais resultados encontrados pelos autores.

\begin{tabular}{|c|c|}
\hline $\begin{array}{l}\text { Autores } \\
\text { (Ano) }\end{array}$ & Principais fatores preditivos e prognósticos \\
\hline $\begin{array}{l}\text { Acle et al. } \\
(2018)\end{array}$ & $\begin{array}{l}\text { Tabagismo, dislipidemia, diabetes mellitus, hiperuricemia, obesidade, alcoolismo, hiper ou hipotireoidismo e } \\
\text { evento cardiovascular prévio estavam presentes no histórico clínico dos pacientes que desenvolveram } \\
\text { síndrome cardiorrenal do tipo 2, fatores como cardiopatia de base ou nefropatia de base também se } \\
\text { configuraram como importantes fatores preditivos. A progressão da doença renal crônica em pacientes com } \\
\text { insuficiência cardíaca com fração de ejeção reduzida está associada a um pior prognóstico em termos de } \\
\text { mortalidade cardiovascular, insuficiência cardíaca e doença cardiovascular, além da própria doença renal. }\end{array}$ \\
\hline $\begin{array}{l}\text { Al-Hwiesh et } \\
\text { al. (2019) }\end{array}$ & $\begin{array}{l}\text { Os principais fatores de risco para o desenvolvimento dessa síndrome são doença renal de base pré-existente, } \\
\text { diabetes mellitus e histórico de insuficiência cardíaca. A piora da função renal após um episódio agudo de } \\
\text { descompensação cardíaca também está associado com o desenvolvimento da síndrome no curto e médio } \\
\text { prazo da hospitalização. Como fatores prognósticos de desfecho clínico, os níveis séricos ou aumentados de } \\
\text { creatinina, de nitrogênio e de ureia plasmáticos configuraram-se como os principais fatores determinantes. A } \\
\text { síndrome cardiorrenal está relacionada a um maior risco do desenvolvimento de doença renal crônica em } \\
\text { estágio terminal. Assim, quanto mais grave o quadro de descompensação, maior o risco de desenvolvimento } \\
\text { dessa injúria. }\end{array}$ \\
\hline $\begin{array}{l}\text { Al-Jarallah et } \\
\text { al. (2018) }\end{array}$ & $\begin{array}{l}\text { Insuficiência cardíaca aguda com diminuição da taxa de filtração glomerular, baixa concentração de } \\
\text { hemoglobina (anemia), hipertensão, doença arterial coronariana, hiperlipidemia, história prévia de } \\
\text { AVC/acidente isquêmico transitório, doença vascular periférica, tabagismo e diabetes configuraram-se como } \\
\text { os principais fatores preditivos para o desenvolvimento de síndrome cardiorrenal aguda. Os pacientes que } \\
\text { desenvolveram essa injúria apresentaram maiores taxas de sangramento, necessidade de transfusão } \\
\text { sanguínea, maior readmissão hospitalar em três meses e de seguimento em até um ano. Os pacientes com } \\
\text { insuficiência cardíaca aguda com fração de ejeção reduzida que desenvolveram síndrome cardiorrenal tipo } 1 \\
\text { estiveram associados com maiores taxas de mortalidade intra-hospitalar. }\end{array}$ \\
\hline $\begin{array}{l}\text { Dos Reis et al. } \\
\text { (2019) }\end{array}$ & $\begin{array}{l}\text { Positividade na dosagem de troponina, cardiomegalia ao raio-x e opacidades intersticiais pulmonares tiveram } \\
\text { maior prevalência nos pacientes com insuficiência cardíaca que desenvolveram síndrome cardiorrenal. } \\
\text { Comorbidades como hipertensão, insuficiência cardíaca crônica, fibrilação atrial, diabetes tipo } 1 \text { ou } 2 \text {, } \\
\text { doença arterial coronariana, disfunção respiratória crônica e doença vascular estiveram presentes na maior } \\
\text { parte dos pacientes que desenvolveram síndrome cardiorrenal. Níveis moderados de disfunção renal estão } \\
\text { associados independentemente com um aumento do risco de mortalidade em pacientes com insuficiência } \\
\text { cardíaca. }\end{array}$ \\
\hline $\begin{array}{l}\text { Inaguma et al. } \\
\text { (2017) }\end{array}$ & $\begin{array}{l}\text { Comorbidades como diabetes mellitus, alto índice de massa corporal, concentrações séricas baixas do } \\
\text { colesterol HDL e evento cardiovascular prévio estiveram relacionadas com maior frequência de eventos } \\
\text { cardiovasculares nos pacientes internados com doença renal crônica. Pacientes com diagnóstico já } \\
\text { estabelecido de insuficiência cardíaca crônica possuem pior prognóstico em relação às disfunções renais e } \\
\text { desfechos clínicos cardiovasculares. }\end{array}$ \\
\hline $\begin{array}{l}\text { Lannemyr et } \\
\text { al. (2018) }\end{array}$ & $\begin{array}{l}\text { O desenvolvimento de disfunção renal em pacientes hospitalizados por insuficiência cardíaca é um forte } \\
\text { preditor para o estabelecimento da síndrome cardiorrenal e configura-se como um forte preditor de } \\
\text { mortalidade e hospitalização quando comparado com a classe funcional da New York Heart Association } \\
\text { (NYHA) e redução da fração de ejeção do ventrículo esquerdo. A elevação da pressão venosa central em }\end{array}$ \\
\hline
\end{tabular}




\begin{tabular}{|c|c|}
\hline & pacientes com insuficiência cardíaca é um importante preditor de disfunção renal. \\
\hline $\begin{array}{l}\text { Pallangyo et } \\
\text { al. (2017) }\end{array}$ & $\begin{array}{l}\text { As comorbidade associadas com o desenvolvimento da síndrome foram doenças hipertensivas do coração, } \\
\text { miocardiopatias, doenças valvares do coração, diabetes e HIV. Os pacientes que desenvolveram síndrome de } \\
\text { anemia cardiorrenal apresentaram maior taxa de mortalidade quando em comparação com os que não } \\
\text { desenvolveram anemia, além de maior predisposição para evolução de disfunção renal em estágio terminal. } \\
\text { Dos preditores de mortalidade mais relevantes, destacaram-se disfunção renal, anemia severa, hiponatremia } \\
\text { e novas hospitalizações. }\end{array}$ \\
\hline
\end{tabular}

Premuzic et al. Os fatores de comorbidades mais comuns identificados foram diabetes, hipertensão, doença arterial (2017) coronariana, doenças valvares do coração e miocardiopatias. A insuficiência cardíaca aguda está associada com maior taxa de mortalidade e hospitalização sendo a disfunção renal um forte e independente fator de risco para a mortalidade desses pacientes. Os fatores prognósticos mais importantes identificados nos pacientes do estudo foram sinais de congestão, disfunção renal e aumento na concentração de creatinina sérica.

Verbrugge et Doença isquêmica do coração, diabetes, classe funcional de NYHA II/III/IV, redução da fração de ejeção do al. (2018) ventrículo esquerdo, diminuição da taxa de filtração glomerular, regurgitação da valva mitral e tricúspide (doenças valvares do coração) e os achados de congestão venosa sistêmica no exame físico estiveram entre os fatores preditivos mais importantes para o desenvolvimento da síndrome cardiorrenal.

Grodin et al. Elevado índice de massa corpórea (IMC), diabetes, Classe funcional NYHA III/IV, redução da fração de (2018) ejeção do ventrículo esquerdo, aumento da pressão venosa na jugular, edema periférico, ascite, diminuição da taxa de filtração glomerular estiveram entre os fatores de risco mais comuns nos pacientes que desenvolveram síndrome cardiorrenal. Pacientes com insuficiência cardíaca aguda que desenvolvem síndrome cardiorrenal estão em alto risco de desfechos clínicos desfavoráveis.

Grossekettler Insuficiência cardíaca congestiva está relacionada com o desenvolvimento de injúria renal crônica, et al. (2019) relacionando-se com a síndrome cardiorrenal e desfechos clínicos ruins. Falha no preenchimento arterial e congestão venosa renal contribuem fortemente para o desenvolvimento do distúrbio. Comorbidades como cardiomiopatia isquêmica, cardiopatia dilatada, hipertensão pulmonar, doenças valvares cardíacas e doença cardíaca hipertensiva foram os fatores preditores mais comuns identificados.

Jung, Hae- Identificaram-se cinco fatores de risco principais entre os pacientes para o desenvolvimento de síndrome Hyuk (2019) cardiorrenal: hipertensão, diabetes, hiperlipidemia, proteinúria e tabagismo.

Li et al. (2018) Infarto agudo do miocárdio e insuficiência cardíaca aguda descompensada foram os fatores mais prevalentes nos pacientes hospitalizados para o desenvolvimento da síndrome cardiorrenal. Dentre as comorbidades mais frequentes, destacaram-se diabetes e hipertensão arterial.

Linhart et al. Inflamação sistêmica induzida por falência cardíaca ou renal em fases agudas com ou sem hipervolemia (2018) pode ser provocada por mecanismos mediados por endotoxinas produzidas pelo próprio organismo, podendo levar à injúria renal. A elevação dos parâmetros pró-inflamatórios (proteína C-reativa, IL-6, procalcitonina, proteína de ligação a lipopolissacarídeo (LBP) encontraram-se elevados nos pacientes com síndrome cardiorrenal aguda e estiveram relacionados com pior prognóstico.

Matsushita et A massa ventricular esquerda está relacionada com a doença renal crônica, enquanto seu tamanho e função al. (2017) estão associados com a albuminúria. Os pacientes que desenvolvem redução na taxa de filtração glomerular e albuminúria estão relacionados com o desenvolvimento de síndrome cardiorrenal. Dentre as comorbidades identificadas nesses pacientes as mais prevalentes foram diabetes, hipertensão, tabagismo, alcoolismo, sedentarismo e histórico de doença arterial coronariana.

Mavrakanas et Diminuição da fração de ejeção do ventrículo esquerdo, aumento da pressão da artéria pulmonar e aumento al. (2017) do diâmetro do ventrículo direito estiveram independentemente associados à maior incidência de síndrome cardiorrenal. Além disso, os pacientes que desenvolveram a forma aguda da síndrome possuem maior risco de progressão para doença renal crônica ou insuficiência cardíaca crônica, além de estar associada com maior taxa de mortalidade. As comorbidades mais prevalentes nesses pacientes foram diabetes, hipertensão, dislipidemia e doença arterial coronariana.

Virzi et al. O aumento nos níveis de citocinas pró-inflamatórias (TNF- $\alpha$, IL-6, IL-18), LPS e marcadores de estresse (2019) $\quad$ oxidativo (mieloperoxidase) em pacientes com síndrome cardiorrenal do tipo 1 estiveram relacionadas com a lesão renal, estado de inflamação no organismo e aumento do estresse oxidativo. As comorbidades mais 


\begin{tabular}{|c|c|}
\hline & comuns encontradas foram diabetes e hipertensão. \\
\hline $\begin{array}{l}\text { Watanabe et } \\
\text { al. (2018) }\end{array}$ & $\begin{array}{l}\text { A elevada relação Sulfato de indoxil/baixa albumina e os seguintes fatores: pressão diastólica, derrame, } \\
\text { fração de ejeção do ventrículo esquerdo estiveram associados com maior mortalidade e pior prognóstico nos } \\
\text { pacientes com insuficiência cardíaca e síndrome cardiorrenal. Dentre as comorbidades mais comuns, } \\
\text { destacam-se hipertensão, diabetes, dislipidemia, tabagismo, doença renal crônica e anemia. }\end{array}$ \\
\hline $\begin{array}{l}\text { Zhang et al. } \\
\text { (2018) }\end{array}$ & $\begin{array}{l}\text { Congestão venosa sistêmica e ativação de mediadores inflamatórios provenientes da injúria renal e cardíaca } \\
\text { desempenham papel fundamental como fatores preditores para o desenvolvimento da síndrome cardiorrenal } \\
\text { em pacientes que apresentam infarto agudo do miocárdio. As comorbidades mais comuns foram diabetes, } \\
\text { hipertensão, dislipidemia, tabagismo, doença renal crônica, doença arterial coronariana, revascularização } \\
\text { percutânea, doença arterial periférica e derrame. }\end{array}$ \\
\hline $\begin{array}{l}\text { García-Blas et } \\
\text { al. (2017) }\end{array}$ & $\begin{array}{l}\text { A exacerbação dos sintomas e o tratamento inadequado da insuficiência cardíaca predispõe os pacientes a } \\
\text { desenvolverem sintomas renais, sendo o agravamento do quadro um importante fator preditor para a } \\
\text { progressão do quadro até o estabelecimento da síndrome cardiorrenal. }\end{array}$ \\
\hline $\begin{array}{l}\mathrm{Ng} \text { et } \\
(2017)\end{array}$ & $\begin{array}{l}\text { Os níveis de proencefalina refletem o estado cardiorrenal e mostraram-se um forte preditor para o } \\
\text { prognóstico de piora da função renal e de mortalidade intra-hospitalar. Dentre o histórico dos pacientes, } \\
\text { fatores como hipertensão, diabetes mellitus, insuficiência cardíaca e renal e doença isquêmica do coração } \\
\text { foram as mais prevalentes entre os pacientes que apresentaram síndrome cardiorrenal. }\end{array}$ \\
\hline $\begin{array}{l}\text { Siegenthaler et } \\
\text { al. (2017) }\end{array}$ & $\begin{array}{l}\text { Os pacientes que apresentaram síndrome cardiorrenal eram mais velhos e possuíam enfermidades como } \\
\text { hipertensão, sinais ecocardiográficos de disfunção diastólica, maiores níveis séricos de pro-BNP e relação } \\
\text { proteinúria/creatinina elevada. }\end{array}$ \\
\hline $\begin{array}{l}\text { Suresh et al. } \\
(2017)\end{array}$ & $\begin{array}{l}\text { Doenças cardiovasculares pré-existentes como doença arterial coronariana, insuficiência cardíaca, arritmias e } \\
\text { morte cardíaca súbito predispõem os indivíduos a desenvolverem síndrome cardiorrenal. Além disso, as } \\
\text { comorbidades mais comuns encontradas nos pacientes foram hipertensão arterial, diabetes mellitus e anemia. }\end{array}$ \\
\hline $\begin{array}{l}\text { Shao et al. } \\
(2018)\end{array}$ & $\begin{array}{l}\text { Idade avançada, sexo masculino, diabetes mellitus, índice de massa corpórea elevado, concentração de ureia } \\
\text { e creatinina séricos elevada, presença de cardiomiopatia (isquêmica, reumática, idiopática ou de outro tipo), } \\
\text { lesões renais causadas por comorbidades tais como doença renal diabética, nefrite glomerular crônica, } \\
\text { glomeruloesclerose hipertensiva e Classe funcional NYHA III e IV, diminuição da taxa de filtração } \\
\text { glomerular, dislipidemias e alterações eletrocardiográficas foram os fatores preditivos mais comuns entre os } \\
\text { pacientes avaliados que desenvolveram a síndrome cardiorrenal devido à insuficiência cardíaca. }\end{array}$ \\
\hline $\begin{array}{l}\text { Robles et al. } \\
(2020)\end{array}$ & $\begin{array}{l}\text { Concentrações séricas elevadas de creatinina plasmática, a redução da taxa de filtração glomerular, cistatina } \\
\text { C, hipercalemia, hemoglobina, pró-BNP e albuminúria estavam presentes nos pacientes que desenvolveram } \\
\text { anemia induzida pela síndrome cardiorrenal. }\end{array}$ \\
\hline
\end{tabular}

Fonte: Autores (2020).

Foi verificado, a partir da análise dos resultados dos artigos selecionados para compor essa pesquisa, que os principais fatores preditivos para o desenvolvimento da síndrome cardiorrenal se associam com os fatores de risco cardiovasculares, tais como hipertensão arterial sistêmica, diabetes mellitus, dislipidemias, tabagismo, doença arterial coronariana, obesidade e às manifestações geradas a partir do comprometimento da função e da perfusão renal.

A partir dos dados obtidos pelos Quadros 3 e 4, foi possível classificar o nível de evidência dos estudos observacionais e ensaios clínicos randomizados pelo sistema GRADE em alto, tendo em vista que os fatores que diminuem o nível de evidência no ECR (limitações metodológicas, inconsistência dos achados, evidência indireta e imprecisão) não foram identificados e os fatores que aumentam nos estudos observacionais (elevada magnitude de efeito, fatores de confusão residuais que aumentam a confiança na estimativa e gradiente dose-resposta) estavam presentes. 
Quadro 3. Avaliação do nível de evidência dos estudos observacionais selecionados para compor essa pesquisa - fatores responsáveis pelo aumento do nível de evidência.

\begin{tabular}{|c|c|c|c|c|}
\hline Autores & $\begin{array}{c}\text { Elevada magnitude de } \\
\text { efeito }\end{array}$ & $\begin{array}{l}\text { Fatores de confusão } \\
\text { residuais que } \\
\text { aumentam a } \\
\text { confiança na } \\
\text { estimativa }\end{array}$ & $\begin{array}{l}\text { Gradiente dose- } \\
\text { resposta }\end{array}$ & $\begin{array}{l}\text { Nível de evidência de } \\
\text { acordo com os } \\
\text { critérios do sistema } \\
\text { GRADE }\end{array}$ \\
\hline Acle et al. (2018) & Presente & Presente & Presente & Alto \\
\hline $\begin{array}{c}\text { Al-Jarallah et al. } \\
\text { (2018) }\end{array}$ & Presente & Presente & Presente & Alto \\
\hline $\begin{array}{l}\text { Dos Reis et al. } \\
\text { (2019) }\end{array}$ & Presente & Presente & Presente & Alto \\
\hline $\begin{array}{c}\text { Inaguma et al. } \\
(2017)\end{array}$ & Presente & Presente & Presente & Alto \\
\hline $\begin{array}{l}\text { Pallangyo et al. } \\
\text { (2017) }\end{array}$ & Presente & Presente & Presente & Alto \\
\hline $\begin{array}{l}\text { Premuzic et al. } \\
\qquad(2017)\end{array}$ & Presente & Presente & Presente & Alto \\
\hline $\begin{array}{l}\text { Verbrugge et al. } \\
\qquad(2018)\end{array}$ & Presente & Presente & Presente & Alto \\
\hline Grodin et al. (2018) & Presente & Presente & Presente & Alto \\
\hline $\begin{array}{c}\text { Grossekettler et al. } \\
\text { (2019) }\end{array}$ & Presente & Presente & Presente & Alto \\
\hline $\begin{array}{c}\text { Jung, Hae-Hyuk } \\
\text { (2019) }\end{array}$ & Presente & Presente & Presente & Alto \\
\hline Li et al. (2018) & Presente & Presente & Presente & Alto \\
\hline Linhart et al. (2018) & Presente & Presente & Presente & Alto \\
\hline $\begin{array}{l}\text { Matsushita et al. } \\
\qquad(2017)\end{array}$ & Presente & Presente & Presente & Alto \\
\hline $\begin{array}{l}\text { Mavrakanas et al. } \\
\qquad(2017)\end{array}$ & Presente & Presente & Presente & Alto \\
\hline Virzi et al. (2019) & Presente & Presente & Presente & Alto \\
\hline $\begin{array}{l}\text { Watanabe et al. } \\
\text { (2018) }\end{array}$ & Presente & Presente & Presente & Alto \\
\hline Zhang et al. (2018) & Presente & Presente & Presente & Alto \\
\hline Ng et al. (2017) & Presente & Presente & Presente & Alto \\
\hline $\begin{array}{c}\text { Siegenthaler et al. } \\
\text { (2017) }\end{array}$ & Presente & Presente & Presente & Alto \\
\hline Suresh et al. (2017) & Presente & Presente & Presente & Alto \\
\hline
\end{tabular}

Fonte: Autores (2020). 
Quadro 3. Avaliação da qualidade dos ensaios cínicos randomizados selecionados para compor essa pesquisa - fatores responsáveis pela diminuição do nível de evidência.

\begin{tabular}{|c|c|c|c|c|c|}
\hline Autores & $\begin{array}{c}\text { Limitações } \\
\text { metodológicas }\end{array}$ & Inconsistência & $\begin{array}{c}\text { Evidência } \\
\text { indireta }\end{array}$ & $\begin{array}{c}\text { Imprecisão } \\
\text { Nível de evidência } \\
\text { de acordo com os } \\
\text { critérios do sistema } \\
\text { GRADE }\end{array}$ \\
\hline $\begin{array}{c}\text { Al-Hwiesh et } \\
\text { al. (2019) }\end{array}$ & Não há & Não há & Não há & Não há & Alto \\
\hline $\begin{array}{c}\text { Lannemyr et } \\
\text { al. (2018) }\end{array}$ & Não há & Não há & Não há & Não há & Alto \\
\hline $\begin{array}{c}\text { García-Blas et } \\
\text { al. (2017) }\end{array}$ & Não há & Não há & Não há & Não há & Alto \\
\hline $\begin{array}{c}\text { Shao et al. } \\
(\mathbf{2 0 1 8 )}\end{array}$ & Não há & Não há & Não há & Não há & Alto \\
\hline $\begin{array}{c}\text { Robles et al. } \\
(\mathbf{2 0 2 0})\end{array}$ & Não há & Não há & Não há & há & \\
\hline
\end{tabular}

Fonte: Autores (2020).

A partir da análise dos Quadros 3 e 4 pode-se inferir que os estudos são caracterizados como estudos observacionais e ensaios clínicos randomizados bem delineados e com achados consistentes.

\section{Discussão}

A síndrome cardiorrenal, condição na qual o comprometimento renal ocorre como resultado da disfunção cardíaca, está associada com maiores riscos de hospitalização e mortes. De certa forma, pode-se afirmar que a mesma é um importante fator preditivo e mais forte que a classe funcional da New York Heart Association (NYHA) ou fração de ejeção reduzida do ventrículo esquerdo. Ainda, pode-se afirmar que pacientes com insuficiência cardíaca que sofrem com deterioração da função renal geralmente desenvolvem síndrome cardiorrenal (Premuzic, Basic-Jukic, Jelakovic \& Kes, 2017; Lannemyr et al., 2018; Pallangyo et al., 2018).

Comorbidades existentes em pacientes com insuficiência cardíaca tais como hipertensão arterial sistêmica, diabetes mellitus, dislipidemias, tabagismo e proteinúria estão associados com desfechos mais críticos e com o desenvolvimento da síndrome cardiorrenal (Mavrakanas, Khattak, Singh \& Charytan, 2017; Jung, 2019).

$\mathrm{Na}$ insuficiência cardíaca aguda descompensada, o desenvolvimento de síndrome cardiorrenal (SCR) está associado com piores desfechos clínicos, hospitalização e maiores gastos com a saúde. O risco de mortalidade associado com a SCR é mais proeminente no início do quadro, mas pode perdurar ao longo da manifestação das consequências clínicas de forma que, quanto mais grave for o quadro de injúria renal, piores serão os possíveis desfechos renais e cardíacos. Distúrbios correlacionados no organismo são compartilhados pelo coração e pelos rins, tais como inflamação e disfunção endotelial celular, resultando em um declínio da função de ambos os órgãos (Al-Hwiesh et al., 2019; Robles et al., 2020).

Dentre seus mecanismos fisiopatológicos, destacam-se os mecanismos hemodinâmicos, levando a uma hipoperfusão renal e redução na taxa de filtração glomerular e os não-hemodinâmicos, como atuação do sistema nervoso simpático e a ativação do sistema renina-angiotensina-aldosterona, inflamação crônica e desequilíbrio entre a produção de espécies reativas de oxigênio e de óxido nítrico. É mais comum em pacientes com síndrome coronariana aguda (Li et al., 2018; Zhang et al., 2018; Virzì et al., 2019). 
Os principais fatores que atuam no mecanismo fisiopatológico da síndrome cardiorrenal são a ativação do sistema renina-angiotensina-aldosterona, sobrecarga de volume, retenção osmótica de sódio, disfunção endotelial, anemia, dislipidemia, coagulopatia e inflamação, todos esses fatores acarretando alterações morfológicas no coração e nos vasos, prejudicando assim a função cardíaca de bombear o sangue e a função renal de filtração, além de ocasionar hipoperfusão renal (Suresh, Arun, Moger \& Swamy 2017).

A relação entre o sistema nervoso simpático e o neuro-hormonal ocorre pela redução da função cardíaca leva à hipoperfusão renal, o que resulta em retenção de sódio para preservar o volume plasmático por meio da ativação do sistema renina-angiotensina-aldosterona (SRAA). O aumento dos níveis de sódio causa a vasoconstricção das arteríolas glomerulares, o que reduz a taxa de filtração glomerular (TFG) (Ng et al., 2017; Siegenthaler et al., 2017; Linhart et al., 2018; Virzì et al., 2019).

No intuito de compensar essa mudança, ocorre o mesmo nas arteríolas eferentes para aumentar a TFG e mantê-la constante. Entretanto, esse mecanismo compensatório agrava ainda mais a hipoperfusão renal e, se muito prolongada, provoca lesão renal em detrimento do estado de hipóxia gerado nos rins. Além disso, o aumento da produção de aldosterona gera aumento da reabsorção de sódio nos túbulos distais renais, gerando uma sobrecarga de volume e aumento do líquido extracelular (García-Blas et al., 2017; Suresh et al., 2017).

A injúria tecidual é causada pelo recrutamento de células inflamatórias e pelo aumento de citocinas, dentre as quais IL-1, IL-6 e TNF- $\alpha$, para a área lesada, além de ser evidenciada por marcadores de estresse oxidativo, como a mieloperoxidase. O aumento de lipopolissacarídeo (LPS) também desempenha função importante, recrutando as citocinas inflamatórias, induzindo inflamação, estresse oxidativo e lesão renal. Essa ativação em longo prazo pode resultar em fibrose tecidual patológica e comprometimento endotelial, agravando a vasoconstricção renal e piora da função cardíaca (Linhart et al., 2018; Virzì et al., 2019).

Parâmetros de inflamação sistêmica estão aumentados em pacientes com síndrome cardiorrenal aguda, independentemente do volume plasmático médio. Na síndrome cardiorrenal, a sobrecarga de volume e congestão venosa são os dois principais mecanismos fisiopatológicos que resultam em inflamação, evento no qual a hiperativação do SRAA e sistema nervoso simpático são os principais fatores desencadeantes (García-Blas et al., 2017; Siegenthaler et al., 2017; Linhart et al., 2018; Zhang et al., 2018).

A presença de hipertensão arterial sistêmica, dislipidemia, diabetes mellitus, ativação do sistema renina-angiotensinaaldosterona, inflamação crônica, estresse oxidativo, resistência à insulina, deficiência de vitamina D e o aumento da concentração de toxinas urêmicas, como sulfato de indoxil, dimetilarginina assimétrica e o fator de crescimento fibroblástico23 (FGF-23) estão associados com a patogênese de doenças cardiovasculares em pacientes com doença renal crônica. O aumento sérico do sulfato de indoxil associado com albumina sérica reduzida está associada com elevada mortalidade, podendo ambos atuarem como fatores preditivos e marcadores prognósticos para doença cardiovascular (Inaguma et al., 2017; Watanabe et al., 2018).

O aumento na pressão venosa central (PVC) é um importante fator preditor de disfunção renal em pacientes com insuficiência cardíaca. A elevação na PVC acarreta uma elevada contrapressão venosa renal, diminuindo a pressão de perfusão renal e prejudicando a função de filtração dos rins (Lannemyr et al., 2018).

Em pacientes com doença renal crônica é muito comum o desenvolvimento de doenças cardiovasculares, tais como arritmias, doença arterial coronariana, insuficiência cardíaca e morte súbita. Esses distúrbios geralmente são os responsáveis pela morbimortalidade nesses pacientes. A proteinúria é considerada um marcador para disfunção endotelial sistêmica e de lesão renal, sendo associada também com mortalidade cardiovascular. Juntamente com a diminuição da taxa de filtração 
glomerular, esses dois fatores atuam como fatores preditivos para o desenvolvimento da síndrome cardiorrenal (Suresh et al., 2017).

O aumento da pressão na doença renal crônica resulta na hipertrofia da parede arterial e em relação parede/lúmen aumentada. Isso acontece por meio do aumento da espessura da parede e do diâmetro arterial devido ao espessamento da camada íntima dos vasos arteriais e deposição de matriz extracelular. Todos esses processos resultam na diminuição da complacência arterial, comprometendo o fluxo sanguíneo do coração a todos os outros órgãos e tecidos. A diminuição da elasticidade arterial é o principal fator responsável pela hipoperfusão do miocárdio, o que eventualmente leva a insuficiência cardíaca crônica (Matsushita et al., 2017).

De acordo com os Guidelines da Kidney Disease: Improving Global Outcomes (KDIGO), em estudo feito em 2011 sobre a doença renal crônica foi verificado que, conforme a taxa de filtração glomerular se deteriorava ou a proteinúria aumentava, a incidência de morte relacionada com doença cardiovascular também se elevava (Inaguma et al., 2017).

Pacientes que desenvolveram anemia induzida por síndrome cardiorrenal foram considerados de categoria de alto risco para mortalidade quando comparados com pacientes sem anemia ou disfunção renal. Essa categoria de alto risco esteve mais relacionada com um preditor independente para readmissão hospitalar devido à insuficiência cardíaca e mortalidade. O desenvolvimento de anemia em pacientes que se apresentam com insuficiência cardíaca e desenvolvem síndrome cardiorrenal e a redução da fração de ejeção do ventrículo esquerdo com disfunção renal podem ser considerados fatores preditivos para o desenvolvimento da síndrome cardiorrenal (Suresh et al., 2017; Al-Jarallah et al., 2019; Robles et al., 2020).

Na síndrome cardiorrenal aguda, a congestão venosa mesentérica provoca edema de parede intestinal, o que permite a translocação de bactérias gram-negativas através das células endoteliais das vilosidades intestinais, com a liberação de LPS na circulação sistêmica e a consequente ativação do sistema imune. A atuação das citocinas inflamatórias, a ativação do sistema imune e o estresse oxidativo desempenham papel fundamental na destruição do epitélio tubular nos rins e das células cardíacas, participando no desenvolvimento de injúria renal aguda e da síndrome cardiorrenal (Virzì et al., 2019).

Os principais fatores de complicação na síndrome cardiorrenal são alterações morfológicas no coração, tais como hipertrofia do ventrículo esquerdo, podendo evoluir eventualmente para redução da fração de ejeção, insuficiência cardíaca congestiva, arritmias e morte súbita, mudanças vasculares, como o espessamento da parede arterial, podendo gerar perda da elasticidade e agravamento da hipertensão arterial, edema agudo de pulmão, devido à hipertensão arterial pulmonar e anemia (Suresh et., 2017).

Um aumento do volume plaquetário médio já foi demonstrado ser um fator preditivo para desfechos clínicos desfavoráveis em pacientes com doença cardiovascular, entretanto, o mesmo ainda não foi demonstrado em pacientes com síndrome cardiorrenal. Tal aumento está relacionado com um aumento na produção e ativação plaquetária, fenômeno que é uma característica marcante na inflamação, podendo ser usado como um biomarcador inflamatório (Li et al., 2018; Zhang et al., 2018).

Plaquetas grandes contêm maior quantidade de citocinas pró-trombóticas e esses fatores possuem variedade de efeitos na função endotelial e na inflamação associada com doença cardiovascular. Ademais, o aumento no volume plaquetário médio é um fator preditor independente de mortalidade em injúria renal aguda (Li et al., 2018; Zhang et al., 2018).

Com o intuito de verificar a existência de correlação entre esses dois fatores, realizou-se uma análise retrospectiva de todos os pacientes com injúria renal aguda que realizaram tratamento com terapia renal substitutiva no Shanghai Sixth People's Hospital, afiliado com a Universidade Jiao Tong de Xangai, na China, ao longo de seis anos. Constatou-se que 35 pacientes desenvolveram síndrome cardiorrenal tipo 1. A partir da análise dos dados, foi possível estabelecer que o aumento no volume plaquetário médio pode ser um fator preditivo para mortalidade intra-hospitalar em curto prazo em pacientes com esse distúrbio (Li et al., 2018). 
Um aumento na relação albumina/creatinina está associada com aumento na massa ventricular esquerda, no tamanho e na função sistólica e diastólica do ventrículo esquerdo. A associação entre albuminúria e alteração estrutural do ventrículo esquerdo pode indicar a presença de albumina na urina como um importante indicador de lesão vascular sistêmica, disfunção endotelial e injúria microvascular. Este último fator desempenha importante papel no desenvolvimento de insuficiência cardíaca. Albuminúria pode também lesionar os rins e estimular a produção de fatores pró-inflamatórios, o que também podem contribuir para alteração da função e estrutura cardíaca (Matsushita et al., 2017; Watanabe et al., 2018).

A progressão da disfunção renal reduz a albumina sérica e deteriora a capacidade de ligação das toxinas urêmicas ligadas a proteínas, dentre as quais, o sulfato de indoxil. O dano celular nas células vasculares humanas resulta de uma resposta pró-inflamatória aumentada e da produção de espécies reativas de oxigênio devido ao aumento sérico do sulfato de indoxil. Acredita-se que esse composto induz disfunção endotelial, promovendo aterosclerose em pacientes com disfunção renal. Quanto maior a relação sulfato de indoxil/redução de albumina nos pacientes, maiores as chances de serem acometidos pelos fatores de risco cardiovasculares (Watanabe et al., 2018).

A partir da análise dos resultados de exames renais, tais como ureia, creatinina, taxa de filtração glomerular, proteinúria, anemia, resultados séricos de albumina, sódio, potássio e cálcio, foi possível verificar uma forte correlação com a piora da função renal em médio prazo em pacientes com insuficiência cardíaca que eram tratados ambulatorialmente. $\mathrm{O}$ principal fator de piora identificado foi a progressiva redução da taxa de filtração glomerular. Os dados apontam que a condição cardíaca não controlada, seja por mudança de estilo de vida associado com tratamento farmacológico, pode estar relacionada com a deterioração da função renal nesses pacientes (García-Blas et al., 2017; Acle, Ormaechea, Álvarez, Gadola \& Noboa, 2018; Zhang et al., 2018).

A proencefalina A (PENK) é amplamente expressa no organismo e as células cardíacas secretam esse pró-hormônio. Em diversas situações clínicas de doenças agudas a concentração elevada no plasma de PENK esteve associada com piora da função renal e pior prognóstico. A concentração plasmática da proencefalina esteve fortemente associada com a taxa de filtração glomerular, sendo um fator preditor importante da piora da função renal e seus níveis aumentaram logo após a lesão renal ( $\mathrm{Ng}$ et al., 2017).

Existem muitos biomarcadores renais e cardíacas que podem ser identificados como forma de detectar injúria nesses órgãos e o quão grave está sendo a lesão. Os principais biomarcadores cardíacos são peptídeo natriurético tipo B (BNP), própeptídeo natriurético tipo B terminal N inativo (NT-proBNP), troponina cardíaca, indoxil sulfato e pró-peptídeo N-terminal de colágeno tipo III (PIIINP). O aumento das concentrações séricas desses biomarcadores indica injúria cardíaca e servem como preditores para o desenvolvimento de insuficiência cardíaca. Enquanto aos biomarcadores de disfunção renal, os principais são os níveis séricos de creatinina e albumina (Inaguma et al., 2017; Watanabe et al., 2018; Zhang et al., 2018).

Ainda sobre os biomarcadores, a associação do natriurético tipo B terminal N inativo (NT-proBNP), da taxa de filtração glomerular estimada e a proteína C-reativa de alta sensibilidade atuam como importantes fatores preditivos na admissão hospitalar de pacientes com insuficiência cardíaca aguda para o desenvolvimento da síndrome cardiorrenal com boa habilidade discriminativa. Esses três biomarcadores juntos mostraram melhor poder preditivo do que individualmente. Pelo menos dois desses elevados estão associados com o desenvolvimento da síndrome cardiorrenal aguda (Ng et al., 2017; Zhang et al., 2018).

Os níveis dos biomarcadores BNP e pro-BNP foram maiores em pacientes com síndrome cardiorrenal do que em pacientes com insuficiência cardíaca aguda com função renal preservada, além de uma maior porcentagem de troponina positiva no mesmo grupo. Esses biomarcadores são importantes para analisar o status de congestão venosa dos pacientes e são recomendados para o manejo de pacientes admitidos com esse tipo de quadro, sendo fundamentais para auxiliar na tomada de decisão clínica e valor prognóstico (Reis et al., 2019). 
Em estudo de coorte prospectivo realizado na França, foi constatado que as admissões nos departamentos de emergência por insuficiência cardíaca aguda estiveram mais comumente relacionadas com comprometimento renal, sendo comum o desenvolvimento de insuficiência renal associada com o quadro cardíaco. A diminuição da função cardíaca leva à redução da perfusão renal, o que pode ser um fator preditivo para insuficiência renal crônica sobrejacente à lesão cardíaca. Assim, o manejo adequado da congestão venosa na emergência parece ter importante valor prognóstico para preservar as funções cardíaca e renal (Reis et al., 2019).

Em estudo de Coorte prospectivo realizado com 1515 pacientes com ou sem histórico de doença arterial coronariana (DAC) em 17 centros de diálise em Toyoake, no Japão, constatou-se que a existência de histórico de DAC em pacientes com doença renal crônica em início de diálise esteve associado com uma maior incidência de eventos de doença cardíaca e maior mortalidade ao longo da diálise. Além disso, distúrbios na função cardíaca podem ser exacerbados com o início da terapia dialítica, tais como hipotensão intra-diálise devido à ultrafiltração (Inaguma et al., 2017).

Em ensaio clínico randomizado realizado com 32 pacientes com insuficiência cardíaca crônica e fração de ejeção reduzida do ventrículo esquerdo $(<40 \%)$ e comprometimento da função renal no Laboratório Clínico Cardíaco Do hospital da Universidade de Sahlgrenska, na Suécia, realizou-se a administração de drogas com propriedades inotrópicas positivas e capacidade de dilatação de artérias e veias, como Levosimendan ou Dobutamina. Constatou-se que ambas as drogas foram capazes de causar um aumento similar no fluxo sanguíneo renal, porém apenas o Levosimendan aumentou a taxa de filtração glomerular além de diminuir com maior eficiência os níveis séricos de creatinina, sendo preferencialmente o agente inotrópico de escolha para tratar pacientes com esse quadro clínico (Lannemyr et al., 2018).

Em estudo de Coorte prospectivo com 463 pacientes com diagnóstico clínico de insuficiência cardíaca (IC) realizado em Dar es Salaam, na Tanzânia, constatou-se que 202 pacientes possuíam a síndrome anêmica cardiorrenal e esta condição esteve associada com o desenvolvimento de doença renal em fase terminal e a forma grave de anemia, com hemoglobina abaixo de 8g/dL, além de estar associada com maiores índices de mortalidade intra-hospitalar em pacientes com IC. A prevalência e a severidade da anemia aumentam conforme ocorre deterioração da função renal. Ademais, hipertensão arterial sistêmica, diabetes mellitus e classe funcional NYHA III ou IV estiveram relacionadas ao desenvolvimento de anemia severa em pacientes com síndrome cardiorrenal (Pallangyo et al., 2018)

Submeteram-se 120 pacientes com insuficiência cardíaca e síndrome cardiorrenal tipo 1 ou 2 em estudo de Coorte prospectivo feito na Croácia, ao longo de 2 anos, a procedimentos de hemofiltração com o intuito de avaliar os benefícios que esse manejo traria aos pacientes. Verificou-se que a hemofiltração veno-venosa contínua em pacientes com insuficiência cardíaca e síndrome cardiorrenal pode modular a resposta inflamatória ao remover as citocinas e toxinas circulantes na corrente sanguínea, além de melhorar a função cardíaca em pacientes com IC. Verificou-se, ainda, que os pacientes tratados com essa modalidade possuíam maior sobrevida (Premuzic et al., 2017).

Em um estudo de coorte retrospectivo realizado com coleta de dados de pacientes submetidos a exame ecocardiográfico a partir do The Research Patient Data Registry (RPDR), que é um banco de dados que reúne informações clínicas de pacientes de 5 instituições de saúde dos Estados Unidos, identificou-se que pacientes que desenvolvem síndrome cardiorrenal frequentemente possuem piores parâmetros ecocardiográficos, sendo isso um fator preditor independente para esse distúrbio. Os três parâmetros mais comumente identificados foram a fração de ejeção do ventrículo esquerdo, pressão arterial pulmonar e o diâmetro do ventrículo direito, que estiveram associados independentemente com síndrome cardiorrenal (Mavrakanas et al., 2017).

Os antagonistas dos receptores de mineralocorticoides (poupadores de potássio), tais como a espironolactona e eplenorona, desempenham papel fundamental em pacientes com insuficiência cardíaca, inibindo o efeito da aldosterona sobre o miocárdio, reduzindo assim a degeneração de fibras, apoptose das células e a fibrose intersticial, prevenindo o remodelamento 
cardíaco. Além disso, são uma classe segura para se usar em IC, mesmo quando há doença renal subjacente. Mesmo em pacientes com alto risco de desenvolvimento da síndrome cardiorrenal, as chances de hipocalemia são baixas (Verbrugge et al., 2018).

Em análise retrospectiva do Estudo de Resgate Cardiorenal em Insuficiência Cardíaca Descompensada Aguda (CARRESS-HF), entre os procedimentos de ultrafiltração e terapia diurética farmacológica, buscou-se ver as principais diferenças entre essas duas modalidades de tratamento. Verificou-se que a ultrafiltração, modalidade de terapia renal substitutiva, mostrou-se mais eficiente em diminuir a congestão quando em comparação com a terapia diurética farmacológica. Esse efeito foi moderado, entretanto, pelas manifestações cardiorrenais e neuro-hormonais ocorridas por causa da rápida remoção de volume, aumento sérico da creatinina, aumento do nitrogênio urêmico no sangue, baixos níveis de sódio e um aumento da atividade da renina durante a ultrafiltração (Grodin et al., 2018).

Diálise peritoneal, outra modalidade de terapia renal substitutiva, está relacionada com uma estabilidade hemodinâmica em pacientes com insuficiência cardíaca. Dentre seus benefícios destacam-se um alívio nas pressões intraabdominais e na pressão venosa renal, além de poder drenar ascites, melhorar a taxa de filtração glomerular, aumentar a diurese e normalizar a creatinina sérica (Shao et al., 2018; Grossekettler et al., 2019).

Esses resultados foram obtidos ao se submeter 159 pacientes com insuficiência cardíaca crônica para tratamento com diálise peritoneal em um estudo de Coorte prospectivo feito na Alemanha. Observou-se, ainda, uma significativa perda de peso nesses pacientes, devido à perda de proteínas causada pelo procedimento, podendo refletir uma perda de massa muscular e até má nutrição e níveis normais de albumina, que se relaciona com uma melhor sobrevida (Grossekettler et al., 2019).

Em insuficiência cardíaca crônica, principalmente cardiomiopatias, há uma elevação sérica nas citocinas circulantes (TNF- $\alpha$ e IL-6) nos tipos dilatados e hipertróficos em resposta ao estresse mecânico e a injúria miocárdica. Esses mediadores pró-inflamatórios são responsáveis pela diminuição na fração de ejeção do ventrículo esquerdo e por um pior prognóstico. A terapia renal substitutiva, dentre as quais a hemofiltração veno-venosa contínua, é capaz de retirar essas citocinas da circulação, além de diminuir solutos e líquido plasmáticos, regulando a pressão e volume sanguíneo e melhorando assim a função cardíaca. As variáveis prognósticas independentes mais importantes são sinais de congestão sistêmica ou pulmonar, disfunção renal e aumento da concentração sérica de creatinina (Premuzic et al., 2017; Virzì et al., 2019).

A ultrafiltração lenta contínua é uma modalidade de terapia renal substitutiva e é uma opção de tratamento para pacientes com insuficiência cardíaca com baixa frequência de admissão hospitalar, porém essa modalidade acarreta um aumento da creatinina sérica após o tratamento. Esse fato não foi observado nos pacientes tratados com hemofiltração venovenosa contínua (Premuzic et al., 2017).

Em relação às limitações encontradas para elaborar este estude destacam-se a inexistência de um protocolo definido e elaborado por instituições de saúde acerca do manejo clínico e tratamento da síndrome cardiorrenal, o que dificultou a coleta das informações mais relevantes e eficientes sobre o tema, outro fator que limitou a pesquisa foi o diagnóstico da síndrome, ainda feita por critérios clínicos e teóricos e não seguida por uma diretriz ou critérios diagnósticos, ainda inexistente.

Dentre as potencialidades desse estudo se sobressaem a heterogeneidade de resultados, de países publicando sobre o tema e a grande quantidade de pacientes analisados nos estudos, fornecendo maior segurança quanto aos achados identificados além de indicar a relevância atual da síndrome cardiorrenal na prática clínica e de apontar a necessidade de uma diretriz (guideline) para um melhor entendimento e maior segurança para a tomada de decisão clínica que favorecerá os pacientes, reduzindo a morbimortalidade e os profissionais da saúde, possibilitando maior segurança na prática médica. 


\section{Considerações Finais}

Levando em consideração todas as manifestações clínicas identificadas no decorrer da síndrome cardiorrenal, o histórico dos pacientes que desenvolveram esse distúrbio, as complicações e os marcadores laboratoriais, pode-se afirmar que os principais fatores preditivos para o desenvolvimento da síndrome cardiorrenal são os fatores de risco para doença cardiovascular, como hipertensão arterial sistêmica, diabetes mellitus, dislipidemias, tabagismo, doença arterial coronariana, insuficiência cardíaca, obesidade e às manifestações geradas a partir do comprometimento da função e da perfusão renal.

Além disso, os marcadores laboratoriais mais comuns que funcionaram como bons fatores preditivos foram a relação albumina/cretina, os biomarcadores de injúria miocárdica além de indicar a lesão cardíaca, também podem indicar a progressão da insuficiência cardíaca até haver comprometimento renal com o desenvolvimento da síndrome cardiorrenal aguda.

É importante ressaltar a relevância desse tema no cenário atual, sendo necessária a elaboração de uma diretriz para o manejo e tratamento da síndrome cardiorrenal, possibilitando a adequada preparação dos profissionais de saúde no intuito de capacitá-los a identificar os sinais preditivos e de evolução para esse distúrbio, tendo como objetivo prevenir a piora da função renal, desfechos clínicos desfavoráveis para os pacientes e a ocorrência de complicações.

Ademais, como apresentado nesse estudo, a síndrome cardiorrenal apresenta uma elevada morbimortalidade e os seus fatores preditivos, identificados nessa revisão, são causas reversíveis e associados à terapêutica relacionada com hipertensão arterial sistêmica, doença arterial coronariana, diabetes mellitus e insuficiência cardíaca. Assim, é essencial ressaltar que o correto manejo clínico dessas comorbidades e acompanhamento deve ser realizado para o controle intensivo desses fatores, podendo prevenir a evolução do curso da doença e evitar o desenvolvimento da síndrome.

No intuito de esclarecer a relação entre os biomarcadores laboratoriais mencionados e a síndrome cardiorrenal, são necessários mais estudos para compreender melhor a associação entre ambos, além de entender as melhores associações farmacológicas para o eficaz manejo dessa condição clínica, objetivando preservar as funções renais e cardíacas.

\section{Referências}

Acle, S., Ormaechea, G., Álvarez, P., Gadola, L., \& Noboa, O. (2018). Función renal en pacientes ambulatorios con insuficiencia cardíaca con fracción de eyección reducida; análisis de su evolución e implicancia pronóstica. Seguimiento a 4 años en una Unidad Multidisciplinaria de Insuficiencia Cardíaca. Revista Uruguaya de Medicina Interna, 3(2), 4-11. http://dx.doi.org/10.26445/03.01.5.

Al-Hwiesh, A. K., Abdul-Rahman, I. S., Al-Audah, N., Al-Hwiesh, A., Al-Harbi, M., Taha, A. \& Al-Audah, N. (2019). Tidal peritoneal dialysis versus ultrafiltration in type 1 cardiorenal syndrome: a prospective randomized study. The International Journal Of Artificial Organs, 42(12), 684-694. http://dx.doi.org/10.1177/0391398819860529.

Al-Jarallah, M., Rajan, R., Al-Zakwani, I., Dashti, R., Bulbanat, B., Sulaiman, K. \& Suwaidi, J. A. (2018). Incidence and impact of cardiorenal anaemia syndrome on all-cause mortality in acute heart failure patients stratified by left ventricular ejection fraction in the Middle East. Esc Heart Failure, 6(1), 103110. http://dx.doi.org/10.1002/ehf2.12351.

Andrade, T. A., Isidorio, U. A., Falcao, K. P. M., Andrade, M. N., Sousa, M. N. A., \& Assis, E. V. (2014). Avaliação da qualidade de vida em pacientes com insuficiência renal crônica. Temas em Saúde (João Pessoa), 14(1), 236-249. http://temasemsaude.com/wp-content/uploads/2015/08/Edicao-01-20141.pdf

Borges Júnior, A. M., Figueiredo, J. P. M. P., \& Sousa, A. B. de. (2020). Complicações apresentadas por pacientes com injúria renal aguda que utilizaram furosemida associada ou não a reposição de albumina. Research, Society and Development, 9(12), e20391211143. https://doi.org/10.33448/rsd-v9i12.11143.

Chimada, C. A., Silva, E. M. da., Otênio, J. K., Pinc, M. M., Jesus, R. A. de, Teixeira, D. G. \&. Zardeto-Sabec, G. (2020). A importância do acompanhamento farmacoterapêutico em pacientes com insuficiência cardíaca. Research, Society and Development, 9(10), e1949108538. https://doi.org/10.33448/rsdv9i10.8538.

Correia, D. M. da S., Cavalcanti, A. C. D., Queluci, G. C., Dias, B. F., Pimentel, A. C. E., Jorge, A. J. L., \&. Rosa, M. L. G. (2020). Prevalência de diagnósticos de enfermagem prioritários em indivíduos segundo fatores de risco para insuficiência cardíaca na atenção primária. Research, Society and Development, 9(10), e9669109351. https://doi.org/10.33448/rsd-v9i10.9351.

Dantas, L. R. C. F., Jesus, C. V. F., Dantas, J. G. L., Martins, B. M., Almeida, A. O., Sousa, A. C. S. \&. Lima, S. O. (2020). Tendências na hospitalização de pacientes com Insuficiência Cardíaca Aguda. Research, Society and Development, 9(9), e725997790. https://doi.org/10.33448/rsd-v9i9.7790.

Galvão, M. C. B., \& Ricarte I. L. M. (2019). Revisão sistemática da literatura: conceituação, produção e publicação. Logeion: Filosofia da Informação, 6(1), 57-73. https://doi.org/10.21728/logeion.2019v6n1.p57-73. 
Galvão T. F., Pansani T. S. A., \& Harrad, D. (2015). Principais itens para relatar Revisões sistemáticas e Meta-análises: a recomendação prisma. Epidemiologia e Serviços de Saúde, 24(2), 335-342. http://dx.doi.org/10.5123/s1679-49742015000200017.

García-Blas, S., Bonanad, C., Llàcer, P., Ventura, S., Nðñez, J. M., Sánchez, R. \&. Vaquer, J. M. (2017). Diuretic Strategies in Acute Heart Failure and Renal Dysfunction: conventional vs carbohydrate antigen 125-guided strategy. clinical trial design. Revista Española de Cardiología (English Edition), 70(12), 1067-1073. http://dx.doi.org/10.1016/j.rec.2017.02.028.

Grodin, J. L., Carter, S., Bart, B. A., Goldsmith, S. R., Drazner, M. H., \& Tang, W. H. W. (2018). Direct comparison of ultrafiltration to pharmacological decongestion in heart failure: a per-protocol analysis of carress-hf. European Journal Of Heart Failure, 20(7), 1148-1156. http://dx.doi.org/10.1002/ejhf.1158.

Grossekettler, L., Schmack, B., Meyer, K., Brockmann, C., Wanninger, R., Kreusser, M. M., \& Katus, H. A. (2019). Peritoneal dialysis as therapeutic option in heart failure patients. Esc Heart Failure, 6(2), 271-279. http://dx.doi.org/10.1002/ehf2.12411.

Inaguma, D., Koide, S., Takahashi, K., Hayashi, H., Hasegawa, M., \& Yuzawa, Y., (2017). Relationship between history of coronary heart disease at dialysis initiation and onset of events associated with heart disease: a propensity-matched analysis of a prospective cohort study. Bmc Nephrology, 18(1), 1-8. http://dx.doi.org/10.1186/s12882-017-0495-8.

Jentzer, J. C., Bihorac, A., Brusca, S. B., Rio-Pertuz, G. del., Kashani, K., Kazory, A. \& Morrow, D. A. (2020). Contemporary Management of Severe Acute Kidney Injury and Refractory Cardiorenal Syndrome. Journal Of The American College Of Cardiology, 76(9), 1084-1101. http://dx.doi.org/10.1016/j.jacc.2020.06.070.

Jung, H. H., (2019). Association of Optimal Blood Pressure With Critical Cardiorenal Events and Mortality in High-Risk and Low-Risk Patients Treated With Antihypertension Medications. Jama Network Open, 2(8), 1-12. http://dx.doi.org/10.1001/jamanetworkopen.2019.9307.

Kumar, U., Wettersten, N., \& Garimella, P. S. (2019). Cardiorenal Syndrome. Cardiology Clinics, 37(3), 251-265. http://dx.doi.org/10.1016/j.ccl.2019.04.001.

Lannemyr, L., Ricksten, S., Rundqvist, B., Andersson, B., Bartfay, S., Ljungman, C., \& Gilljam, T. (2018). Differential Effects of Levosimendan and Dobutamine on Glomerular Filtration Rate in Patients With Heart Failure and Renal Impairment: a randomized double : blind controlled trial. Journal Of The American Heart Association, 7(16), 1-9. http://dx.doi.org/10.1161/jaha.117.008455.

Legrand, M., Berardinis, B. de, Gaggin, H. K., Magrini, L., Belcher, A., Zancla, B., \& Sambhare, R. (2014). Evidence of Uncoupling between Renal Dysfunction and Injury in Cardiorenal Syndrome: insights from the bionics study. Plos One, 9(11), 1-8. http://dx.doi.org/10.1371/journal.pone.0112313.

Li, J., Sheng, X., Cheng, D., Wang, F., Jian, G., Li, Y. \& Wang, N. (2018) Is the mean platelet volume a predictive marker of a high in-hospital mortality of acute cardiorenal syndrome patients receiving continuous renal replacement therapy? Medicine, 97(25), 1-5. http://dx.doi.org/10.1097/md.0000000000011180.

Linhart, C., Ulrich, C., Greinert, D., Dambeck, S., Wienke, A., Girndt, M. \& Pliquett, R. U. (2018). Systemic inflammation in acute cardiorenal syndrome: an observational pilot study. Esc Heart Failure, 5(5), 920-930. http://dx.doi.org/10.1002/ehf2.12327.

Lullo, L. di, Bellasi, A., Barbera, V., Russo, D., Russo, L., Iorio, B., \& Ronco, C. (2017). Pathophysiology of the cardio-renal syndromes types 1-5: an uptodate. Indian Heart Journal, 69(2), 255-265. http://dx.doi.org/10.1016/j.ihj.2017.01.005.

Matsushita, K., Kwak, L., Sang, Y., Ballew, S. H., Skali, H., Shah, A. M., \& Solomon, S. (2017). Kidney Disease Measures and Left Ventricular Structure and Function: the atherosclerosis risk in communities study. Journal Of The American Heart Association, 6(9), 1-20. http://dx.doi.org/10.1161/jaha.117.006259.

Mavrakanas, T. A., Khattak, A., Singh, K., \& Charytan, D. M. (2017). Epidemiology and Natural History of the Cardiorenal Syndromes in a Cohort with Echocardiography. Clinical Journal Of The American Society Of Nephrology, 12(10), 1624-1633. http://dx.doi.org/10.2215/cjn.04020417.

Melo, W, F., Bezerra, A. L. D., \& Sousa, M. N. A. (2014). Perfil epidemiológico dos pacientes com insuficiência renal crônica: um estudo quantitativo. Ciência \& Desenvolvimento-Revista Eletrônica da FAINOR, 7(2), 142 -156.

Ministério da Saúde. Secretaria de Ciência, Tecnologia e Insumos Estratégicos. Departamento de Ciência e Tecnologia. Diretrizes metodológicas: Sistema GRADE - Manual de graduação da qualidade da evidência e força de recomendação para tomada de decisão em saúde: Ministério da Saúde; 2014. http://bvsms.saude.gov.br/bvs/publicacoes/diretrizes_metodologicas_sistema_grade.pdf

Ng, L. L., Squire, I. B., Jones, D. J. L., Cao, T. H., Chan, D. C. S., Sandhu, J. K., \&Hartmann, O. (2017). Proenkephalin, Renal Dysfunction, and Prognosis in Patients With Acute Heart Failure. Journal Of The American College Of Cardiology, 69(1), 56-69. http://dx.doi.org/10.1016/j.jacc.2016.10.038.

Pallangyo, P., Fredrick, F., Bhalia, S., Nicholaus, P., Kisenge, P., Mtinangi, B., \& Humphrey, S. (2017). Cardiorenal Anemia Syndrome and Survival among Heart Failure Patients in Tanzania: a prospective cohort study. Bmc Cardiovascular Disorders, 17(1), 1-6. http://dx.doi.org/10.1186/s12872-017-0497-2.

Premuzic, V., Basic-Jukic, N., Jelakovic, B., \& Kes, P. (2017). Continuous Veno-Venous Hemofiltration Improves Survival of Patients With Congestive Heart Failure and Cardiorenal Syndrome Compared to Slow Continuous Ultrafiltration. Therapeutic Apheresis And Dialysis, $21(3)$, 279-286. http://dx.doi.org/10.1111/1744-9987.12516.

Raina, R., Nair, N., Chakraborty, R., Nemer, L., Dasgupta, R., \& Varian, K. (2020). An Update on the Pathophysiology and Treatment of Cardiorenal Syndrome. Cardiology Research, 11(2), 76-88. http://dx.doi.org/10.14740/cr955.

Rangaswami, J., \& Mathew, Roy O. (2018). Pathophysiological Mechanisms in Cardiorenal Syndrome. Advances In Chronic Kidney Disease, 25(5), 400-407. http://dx.doi.org/10.1053/j.ackd.2018.08.006.

Reis, D. dos, Fraticelli, L., Bassand, A., Manzo-Silberman, S., Peschanski, N., Charpentier, S. \& Laribi, S. (2019). Impact of renal dysfunction on the management and outcome of acute heart failure: results from the french prospective, multicentre, defssica survey. Bmj Open, 9(1), 1-11. http://dx.doi.org/10.1136/bmjopen-2018-022776. 
Research, Society and Development, v. 10, n. 2, e3410212090, 2021

(CC BY 4.0) | ISSN 2525-3409 | DOI: http://dx.doi.org/10.33448/rsd-v10i2.12090

Robles, N. R., Campillejo, R. D., Valladares, J., Vinuesa, E. G. de, Villa, J., \& Gervasini, G. (2020). Sacubitril-valsartan Improves Anemia of Cardiorenal Syndrome (CRS), Cardiovascular \& Hematological Agents in Medicinal Chemistry, 18(X). https://doi.org/10.2174/1871525718666200506095537.

Savira, F., Magaye, R., Liew, D., Reid, C., Kelly, D. J., Kompa, A. R., \& Wang, B. H. (2020). Cardiorenal syndrome: multi : organ dysfunction involving the heart, kidney and vasculature. British Journal Of Pharmacology, 177(13), 2906-2922. http://dx.doi.org/10.1111/bph.15065.

Shao, Q., Xia, Y., Zhao, M., Liu, J., Zhang, Q., Jin, B., \& Jiang, C. (2018). Effectiveness and Safety of Peritoneal Dialysis Treatment in Patients with Refractory Congestive Heart Failure due to Chronic Cardiorenal Syndrome. Biomed Research International, 2018(X), 1-9. http://dx.doi.org/10.1155/2018/6529283.

Siddaway, A. P., Wood, A. M., \& Hedges, L. V. (2019). How to Do a Systematic Review: a best practice guide for conducting and reporting narrative reviews, meta-analyses, and meta-syntheses. Annual Review Of Psychology, 70(1), 747-770. http://dx.doi.org/10.1146/annurev-psych-010418-102803.

Siegenthaler, M., Huynh-Do, U., Krayenbuehl, P., Pollock, E., Widmer, U., Debaix, H., \& Ruschitzka, F. (2017). Impact of cardio-renal syndrome on adverse outcomes in patients with Fabry disease in a long-term follow-up. International Journal Of Cardiology, $249(\mathrm{X}), 261-267$. http://dx.doi.org/10.1016/j.ijcard.2017.09.027.

Suresh, H., Arun, B. S., Moger, V., \& Swamy, M. (2017). Cardiorenal syndrome type 4: a study of cardiovascular diseases in chronic kidney disease. Indian Heart Journal, 69(1), 11-16. http://dx.doi.org/10.1016/j.ihj.2016.07.006.

Uduman, Jr. (2018). Epidemiology of Cardiorenal Syndrome. Advances In Chronic Kidney Disease, 25(5), 391-399. http://dx.doi.org/10.1053/j.ackd.2018.08.009.

Verbrugge, F. H., Martens, P., Ameloot, K., Haemels, V., Penders, J., Dupont, M., \& Mullens, W. (2018). Spironolactone to increase natriuresis in congestive heart failure with cardiorenal syndrome. Acta Cardiologica, 74(2), 100-107. http://dx.doi.org/10.1080/00015385.2018.1455947.

Virzì, G. M., Breglia, A., Castellani, C., Ankawi, G., Bolin, C., Cal, M. de., \& Ronco, C. (2019). Lipopolysaccharide in systemic circulation induces activation of inflammatory response and oxidative stress in cardiorenal syndrome type 1. Journal Of Nephrology, 32(5), 803-810. http://dx.doi.org/10.1007/s40620-01900613-2.

Watanabe, I., Tatebe, J., Fujii, T., Noike, R., Saito, D., Koike, H., \& Amano, H. (2019). Prognostic Significance of Serum Indoxyl Sulfate and Albumin for Patients with Cardiovascular Disease. International Heart Journal, 60(1), 129-135. http://dx.doi.org/10.1536/ihj.18-116.

Yogasundaram, H., Chappell, M. C., Braam, B., \& Oudit, G. Y. (2019). Cardiorenal Syndrome and Heart Failure-Challenges and Opportunities. Canadian Journal Of Cardiology, 35(9), 1208-1219. http://dx.doi.org/10.1016/j.cjca.2019.04.002.

Zhang, D., Li, H., Chen, H., Ma, Q., Chen, H., Xing, Y., \& Zhao, X. (2018) Combination of Amino-Terminal Pro-BNP, Estimated GFR, and High-Sensitivity CRP for Predicting Cardiorenal Syndrome Type 1 in Acute Myocardial Infarction Patients. Journal Of The American Heart Association, 7(19), 1-11. http://dx.doi.org/10.1161/jaha.118.009162. 